\title{
COVID-19: All Facts, No Myth
}

\author{
Panday DR, ${ }^{1}$ Panday KR, ${ }^{2}$ Rauniar GP ${ }^{1}$
}

${ }^{1}$ Department of Clinical Pharmacology and

Therapeutics,

${ }^{2}$ Department of Basic and Clinical Physiology

BP Koirala Institute of Health Sciences

Dharan, Nepal.

\section{Corresponding Author}

Dipesh Raj Panday

Department of Clinical Pharmacology and Therapeutics,

BP Koirala Institute of Health Sciences

Dharan, Nepal.

E-mail: dipesh.pandey@bpkihs.edu

\section{Citation}

Panday DR, Panday KR, Rauniar GP. COVID-19, All Facts, No Myth. Kathmandu Univ Med J. 2020;COVID-19 Special Issue 70(2):80-9.

\begin{abstract}
On December 31, 2019, the China Health Authority alerted WHO about 27 cases of pneumonia of unknown etiology in Wuhan City. It was subsequently named Severe Acute Respiratory Syndrome Coronavirus-2 (SARS-CoV-2) and the disease as Coronavirus Disease 2019 (COVID-19). The disease has now become pandemic. Current review was done to summarize information on COVID-19 published in various scientific works.
\end{abstract}

Electronic databases containing medical articles viz., MEDLINE/PubMed, Google Scholar etc were searched using the Medical Subject Headings 'COVID-19', '2019nCoV', 'coronavirus' and 'SARS-CoV-2' during antecedent one year. All study designs were incorporated to harvest clinical, laboratory, imaging, and hospital course data.

The intermediate host of the virus is still unknown. Respiratory droplets produced by the patient is main source of transmission. SARS-CoV-2 invades the airway epithelium by binding to angiotensin-converting enzyme-2 (ACE2) receptor with Coronavirus spike (S) protein. Most common symptoms are fever (98\%), dry cough (77\%), and dyspnea (63.5\%). Later, complications like acute respiratory distress syndrome, septic shock etc may occur. Advanced age and co-morbidities like Diabetes have higher mortality otherwise Case Fatality Rate is 2-3\%. RT$P C R$ is the diagnosis of choice. Since no universally accepted registered drug or FDA approved vaccine has come by now, prevention is the key. Hands should be regularly cleaned with soap or alcohol based sanitizer and in public, Nose and Mouth should be covered with face-mask and social distance of one meter should be maintained. While Vaccines are expected by early 2021, we should not forget to take comprehensive measures to prevent future outbreaks of zoonotic origin.

\section{KEY WORDS}

2019-nCoV, COVID-19, Pandemic, SARS-CoV-2

\section{INTRODUCTION}

On December 31, 2019, the China Health Authority alerted the World Health Organization (WHO) about 27 cases of pneumonia of unknown etiology in Wuhan City of Hubei Province in Central China. ${ }^{1}$ Few days prior to that, the first case of the disease was already diagnosed on December 12 , 2019. Many of these cases, had a common exposure to the Hunan wholesale seafood market that also traded live animals. WHO recruited surveillance system (originally came into existence during the SARS outbreak) that collected throat swabs of the patient and sent them to reference labs for etiologic investigations. ${ }^{2}$ On $7^{\text {th }}$ January, it was identified to be a Coronavirus that had more than $95 \%$ homology with the bat Coronavirus and $>70 \%$ similarity with the Severe Acute Respiratory Syndrome Corona Virus
(SARSCoV) detected in China on 2003. ${ }^{3}$ Environmental samples from the Hunan seafood market also tested positive, signifying that the virus originated from there. So the Hunan sea food market was closed. ${ }^{4}$

Etiological agent was first named 2019-novel-Corona Virus (2019-nCoV) by WHO and later renamed as Severe Acute Respiratory Syndrome Coronavirus-2 (SARS-CoV-2) by the Coronavirus Study Group and the disease was named Coronavirus Disease 2019 (COVID-19) by the WHO. ${ }^{5}$

The first fatal case was reported on $11^{\text {th }}$ January 2020. By $23^{\text {rd }}$ January, the 11 million population of Wuhan was placed under lock-down with restrictions of entry and exit from the region. It was presumed that the massive 
migration of Chinese during the Chinese New Year during that period fuelled the epidemic. Soon this lock-down was extended to other cities of Hubei province. Later, WHO declared the SARS-CoV-2 outbreak as pandemic or Public Health Emergency of International Concern (PHEIC) on January 30, 2020. ${ }^{6}$ Later, cases of COVID-19 were detected even in countries having no direct history of travel to China, suggesting that local human-to-human transmission is possible. ${ }^{7}$

Due to the rapid mutation and genetic recombination of $\mathrm{CoV}$, there have been two events in the past two decades wherein crossover of animal beta-corona viruses to humans resulted in severe diseases. The first such instance was in 2002-2003 when a new Coronavirus of the $\beta$ genera and with origin in bats, crossed over to humans via the intermediary host of palm civet cats in the Guangdong province of China. The virus was designated as SARS Coronavirus and it affected 8422 people mostly in China and Hong Kong and caused 916 deaths with mortality rate being $11 \%$ before it was finally contained. ${ }^{8}$

Almost a decade later in 2012, MERS-CoV, also of bat origin, emerged in Saudi Arabia with dromedary camels as the intermediate host and affected 2494 people and caused 858 deaths (fatality rate being $34 \%$ ). ${ }^{9}$

Recombination rates of CoVs are very high because of constantly developing transcription errors and RNA Dependent RNA Polymerase (RdRP) jumps. ${ }^{9}$ With its high mutation rate, Coronaviruses are zoonotic pathogens that are present in humans and various animals with a wide range of clinical features from asymptomatic course to requirement of hospitalization in the intensive care unit; causing infections in respiratory, gastrointestinal, hepatic and neurologic systems.

This is an authors' endeavor to summarize facts regarding COVID-19 published in different scientific literatures and replace the chaos brought by anecdotal claims with scientific knowledge.

\section{METHODS}

Electronic databases MEDLINE/PubMed, Google Scholar, WHO COVID-19 database, Excerpta Medica/EMBASE, Scopus, Thomson Reuters' Web of Science, Scopus, UpToDate, CINAHL, Cochrane Database of Systematic Reviews and Database of Abstracts and Reviews of Effectiveness (DARE), Asian Science Citation Index (ASCI), IranMedex, Scientific Information Database (SID), Index Medicus for the World Health Organization, Eastern Mediterranean Region (IMEMR) and other regions, PakMediNet, IndMed, etc were searched using the Medical Subject Headings terms "COVID-19', '2019-nCoV', 'coronavirus' and 'SARS-CoV-2' during antecedent one year. We limited our search results to publications in English and Chinese (using Google Translate) and excluded abstracts from conferences and commentaries. We accepted the following types of studies: cross-sectional, cases series, case-control, case reports, review articles, opinion papers, and letters to journal editors that incorporated clinical, laboratory, imaging, and hospital course of patients.

\section{DISCUSSION}

\section{Virus classification and Structure}

According to the PREDICT, an epidemiological research program funded by a United States Agency for International Development (USAID) grant, there are almost 1.6 million unknown species of viruses found in avian and mammalian populations, out of which 700,000 viral species have the potential to infect humans. ${ }^{7}$ Coronaviruses (CoV) are a large family of spherical or pleomorphic, crown-shaped, enveloped positive sense single-stranded RNA viruses that belong to the order Nidovirales, family Coronaviridae, subfamily Coronavirinae. The subfamily Coronavirinae are subdivided into four genera:

(a) Alpha coronavirus contains the human Coronavirus (HCoV)-229E and HCoV-NL63;

(b) Beta coronavirus includes HCoV-OC43, SARS-CoV, CoVHKU1, and Middle East Respiratory Syndrome Coronavirus (MERS-CoV);

(c) Gamma coronavirus includes viruses of whales and birds

(d) Delta coronavirus includes viruses isolated from pigs and birds.

Phylogenetic analysis of the SARS-CoV-2 genome indicates that the virus is closely related (with $88 \%$ identity) to two bat-derived SARS-like Coronaviruses collected in 2018 in eastern China (bat-SL-CoVZC45 and bat-SL-CoVZXC21) and genetically distinct from SARS-CoV (with about 79\% similarity) and MERS-CoV. ${ }^{10}$

Cryo-electron microscopic and tomographic technique shows that Coronaviruses range $60 \mathrm{~nm}$ to $140 \mathrm{~nm}$ in diameter with spike (club-shaped glycoprotein) like projections on its surface giving it a Crown like appearance under the electron microscope; hence the name Coronavirus. Genome changes results from recombination, gene exchange, gene insertion, or deletion. ${ }^{11,12}$

The virions have four main structural proteins namely the spike, membrane, envelope and nucleocapsid. A fifth structural protein namely the hemagglutinin esterase is also present in the subset of $\beta$-Coronaviruses which binds sialic acids on the surface glycoprotein and have acetylesterase activity. ${ }^{13}$

\section{Steps of infection of host by Corona virus}

The life cycle of a Coronavirus is subdivided into four phases: ${ }^{14}$

(i) Entry and Attachment

(ii) Replicase Protein Expression 
(iii) Replication and Transcription

(iv) Assemble and Release

\section{Epidemiology Corona Viruses}

Regarding both SARS-CoV and MERS-CoV, bats served as the natural reservoir and humans the terminal host, with the palm civet and dromedary camel respectively being intermediary hosts. ${ }^{15,16}$ Whole genome sequencing showed that bats are the host for this virus as the homology between SARS-CoV-2 and bat Coronaviruses is $96 \% .{ }^{11}$ Regrettably, the intermediate host for SARS-CoV-2 is still unknown. It is because when the epidemic was first reported in Wuhan in December 2019, most of the bat species were hibernating and no bats were being sold or found at the Hunan seafood market; however, various non-aquatic animals (including mammals) were being sold. It is suggested that snakes or Pangolins might be the wildlife animal reservoir, which acted as intermediate host for the SARS-CoV-2. However, it is not yet confirmed that which wildlife animal is exactly responsible for transmitting SARS-CoV-2 to humans, and further investigations are still required. ${ }^{17}$ Nevertheless, bats do not always need an intermediary host to transmit viruses to humans. For example, Nipah virus in Bangladesh is transmitted through bats shedding into raw date palm sap. $^{18}$

The number of cases of COVID-19 escalated exponentially, most of which did not have exposure to the live animal market, suggestive of the fact that human-to-human transmission does occur. ${ }^{4}$ Clusters of infected family members and medical workers have confirmed the presence of person-to-person transmission. ${ }^{19}$ The timing of the disease coincided with the most famous traditional festival in China, during which nearly 3 billion people travelled countrywide. It might have been responsible for the dissemination of virus.

\section{Modes of Transmission}

Person-to-person transmission is thought to occur among close contacts mainly via respiratory droplets produced when an infected person coughs or sneezes. Fomites may be another large source of transmission, as SARS-CoV has been found to persist on surfaces upto 96 and other Coronaviruses for up to 9 days. Thus, their mode of spread is just like the spread of influenza and other respiratory pathogens. When compared to H1N1 influenza, which shared the same transmission pathways, the cumulative attack rate of COVID-19 is 50-fold higher. ${ }^{19}$ Infection is acquired either by inhalation of these droplets or touching surfaces contaminated by them and then touching the mouth, nose, or possibly eyes. ${ }^{20}$ Whether or not there is asymptomatic transmission of disease is controversial. ${ }^{7}$ The virus is destroyed in less than a minute by Surface disinfection using $71 \%$ ethanol, $0.5 \%$ hydrogen peroxide or $0.1 \%$ sodium hypochlorite. ${ }^{21}$
A study indicated the mean incubation period was 5.2 days (95\% confidence interval [95\% Cl]: 4.1-7.0). In yet another studies, the incubation period has been found to be as long as 19 or 24 days. ${ }^{22}$ The basic reproductive number (BCR/ R0), defined as average number of individuals an affected person infects, for this disease is around 2.9 or 2.2 (95\% $\mathrm{Cl}$ : 1.4-3.9). ${ }^{22}$ However, later in a further analysis of 12 available studies, it was found that RO for this disease is 3.28 with range being 2 to $6.47 .{ }^{23}$ In comparison, the BCR of SARS was 2 and 1.3 for pandemic flu H1N1. It means this disease is more contagious in comparison. The greatest risk in COVID-19 is transmission to healthcare workers. In SARS outbreak of 2002, $21 \%$ of those affected were healthcare workers. ${ }^{24}$

\section{Pathogenesis and immune response}

The released SARS-CoV-2 viruses infect kidney cells, liver cells and T lymphocytes, as well as the lower respiratory tract, where they form the main symptoms and signs. SARS-CoV-2 binds to the angiotensin-converting enzyme-2 (ACE2) receptor through the Coronavirus spike (S) protein to invade airway epithelium and alveolar type 2 (AT2) pneumocytes and pulmonary cells that normally synthesize pulmonary surfactant. This promotes direct toxicity and excessive immune responses which stimulates a systemic inflammatory response, forming a cytokine storm, that leads to lung tissue injury. In severe cases, the disease can lead to complications like acute respiratory distress syndrome, septic shock, metabolic acidosis, coagulation dysfunction, and multiple organ dysfunction syndromes and death. Individuals with reduced immunity and patients with comorbidities are highly susceptible..$^{25,26}$

\section{Risk factors}

SARS-CoV usually infects young people, MERS-CoV attacks people aging above 50 years and COVID-19 infects people of middle age and above. There is no evidence for intrauterine infection caused by vertical transmission of SARS-CoV-2. ${ }^{27}$ Again, disease in neonates, infants and children have been also reported to be significantly milder than their adult counterparts. The clinical features of infected pediatric patients vary, but most have had mild symptoms with no fever or pneumonia, and have a good prognosis. Children might be less likely to be infected or, if infected, present milder manifestations than adults; therefore, it is possible that their parents will not seek out treatment leading to underestimate of COVID-19 incidence in this age group. ${ }^{28,29}$

The incidence of SARS-CoV-2 infection is seen most often in adult male patients with the median age being 34 to 59 years. The highest proportion of severe cases occurs in adults $\geq 60$ years of age, and in those with certain underlying conditions, such as cardio-vascular and cerebrovascular diseases and diabetes. Adverse outcomes and death are more common in the elderly and those with underlying co-morbidities (50-75\% of fatal cases). There 
is an anecdotal predisposition of the Asian population to the virus due to higher expression of ACE2 receptors on the respiratory mucosa. ${ }^{30}$

\section{Clinical manifestations and complications}

Clinical manifestations of SARS-CoV-2 infection are similar to SARS-CoV. Approximately, 50-75\% of individuals with positive Real-time Reverse Transcriptase Polymerase Chain Reaction (RT-PCR) throat swab remain asymptomatic, whilst others develop mild flu-like symptoms and a further small percentage (about $10 \%$ of all symptomatic patients) present dyspnoea, severe interstitial pneumonia, ARDS and multiorgan dysfunction. The most common and frequently reported symptoms of COVID-19 are fever (98\%), dry cough (77\%), and dyspnea (63.5\%) whereas less common symptoms are myalgia, malaise, arthralgia, chest pain, nasal congestion, runny nose, headache, sore throat, sputum production and even diarrhea. ${ }^{31,32}$ Fever, cough, shortness of breath are also often the first symptoms developed by patients. ${ }^{33}$ The hyposmia and dysgeusia are often reported by the patients. These indicate the neurotropism of the virus which invades the olfactory nerve, bulb and alternatively, the sensory fibres of the vagus nerve from the brainstem which innervates different organs of the respiratory tract, including the larynx, trachea and lungs. ${ }^{34}$

Approximately $75 \%$ patients in due course develop bilateral pneumonia, which later turn into bilateral multiple lobular and sub-segmental consolidation followed by ARDS, secondary infections, septic shock, refractory metabolic acidosis, coagulation disorder, and MODS. ${ }^{19}$ Other features are acute heart damage and pneumothorax, arrhythmia, acute kidney injury and even intestinal symptoms.

Different from SARS-CoV and MERS-CoV infections, very few COVID-19 patients show prominent upper respiratory tract signs and symptoms such as rhinorrhoea, sneezing, or sore throat, suggesting that the virus might have greater preference for lower respiratory tract. ${ }^{4}$ The median time from onset of symptoms to dyspnea is 5 days, to hospitalization is 7 days and to acute respiratory distress syndrome (ARDS) is 8 days. ${ }^{32}$

The course of the infection is mild or asymptomatic in about $90 \%$ of cases. It becomes serious only in around $10 \%$ of cases, with dyspnoea, hypoxaemia and extensive radiological involvement of the lung parenchyma. A critical condition develops in around $5 \%$ of cases, with respiratory failure, pneumonia, shock, multiorgan failure and, in the most serious cases, death, which is almost always caused by progression to ARDS to multiorgan failure. ${ }^{35,36}$ The mortality rate is variable, ranging from $2 \%$ to $5 \%$, however it depends upon age-group, co-morbidity, and may even be geography and gene. ${ }^{37}$

\section{Diagnosis}

As per recommendations of World Health Organization (WHO) and Centers for Disease Control and Prevention
$(C D C)$, Specific diagnosis is by specific molecular tests (Realtime Reverse Transcriptase Polymerase Chain Reaction) on respiratory samples (Throat Swab/ Nasopharyngeal Swab/ Endotracheal Aspirates And Bronchoalveolar Lavage). The sensitivity rate is not clear but is estimated to be around $66-80 \%$. The specificity of the RT-PCR test seems to be very high. ${ }^{38}$

Positive pharyngeal swab remains for several weeks after remission of symptoms. ${ }^{39}$

The sensitivity of antibody detection is generally lower than molecular methods and is mostly used in retrospective diagnosis. However, commercial tests are not available as yet. $^{40}$

Computed tomography findings are important for both diagnosis and follow-up. ${ }^{41}$

Viral culture is more time consuming. viral cultures can be used in the in-vitro and in-vivo antiviral therapy and vaccine evaluation trials. ${ }^{42}$

\section{Case definitions ${ }^{43}$}

\section{Suspected COVID-19 case}

A patient having a severe acute respiratory symptoms like fever, cough but no alternate diagnosis be firmly ascertained, with a positive history of travel* to or residence in a country/area or territory reporting local transmission of COVID-19 within antecedent 14 days of symptoms onset.

*travel history becomes important. However, as the epidemic has spread, the travel history will become irrelevant.

\section{OR}

A patient with an acute respiratory illness AND having been in contact with a confirmed or probable COVID-19 case in the last 14 days prior to the onset of symptoms.

OR

A patient with a severe acute respiratory infection (fever and at least one sign/symptom of respiratory disease (e.g., cough, shortness breath) AND requiring hospitalization and with no other etiology that fully explains the clinical presentation.

\section{Probable case}

If a suspect case for whom testing for COVID-19 is inconclusive.

\section{OR}

A suspect case for whom testing could not be performed for any reason.

\section{Confirmed case}

Person with laboratory confirmation of SARS-CoV-2, irrespective of clinical signs and symptoms. 


\section{Other Laboratory findings}

Among COVID-19 patients, common laboratory abnormalities include. ${ }^{32}$

Leukopenia (in 9-25\%), Leukocytosis (in 24-30\%), Thrombocytopenia in about $12 \%$ and Lymphocytopenia in about 63\%. Same studies also showed Increased Neutrophil-To-Lymphocyte Ratio (NLR), Prolonged Prothrombin Time, Elevated Lactate Dehydrogenase. Other studies showed elevated ESR (in around 84\%) and increased C-Reactive Protein, elevated Creatinine and increased ALT/AST ratio. ${ }^{4,44-46}$ Similarly, D-dimer, CPK and LDH all are often elevated and high levels are associated with severe disease. Similarly, procalcitonin level is usually normal. However, a high level indicates a bacterial coinfection. ${ }^{29}$ Further, elevated Troponin level appears to be a strong prognostic indicator of mortality. ${ }^{47}$

\section{Radiological findings}

Chest X-ray and CT findings usually show bilateral infiltrates as well as unilateral involvement but it may be normal in early disease. ${ }^{43}$ Typical X-ray findings of COVID-19 lungs in children is patchy ground-glass opacities. ${ }^{48} \mathrm{CT}$ imaging generally shows infiltrates, ground opacities and subsegmental consolidation. Chest CT has high diagnostic value even in patients who have negative Reverse Transcription-Polymerase Chain Reaction (RT-PCR). Many CT positive cases, which are initially molecular tests negative, become positive in due course of time. Infact, in experts' hands, CT is more sensitive and specific than molecular techniques. Therefore, CT scans have also been used to diagnose COVID-19 in suspect cases with negative molecular diagnosis. ${ }^{49}$

\section{Case Fatality Rate (CFR)}

COVID-19 is being considered as devastating as World influenza epidemic of 1918. Virus spreads faster than its two ancestors the SARS-CoV and MERS-CoV, but has lower fatality. ${ }^{14}$

MERS-CoV caused 858 fatalities since September 2012. 2494 laboratory-confirmed cases of infection were detected in 27 countries making MERS-CoV's CFR=34.40\%. The outbreak got controlled in 2013, although sporadic cases continued to be reported throughout the rest of the year. Similarly, SARS-CoV had $35.6 \%$ mortality rate. Despite much higher CFRs for SARS (9.6\%) and MERS (34.4\%), COVID-19 has led to more total deaths due to the large number of cases. ${ }^{50}$

The CFR of COVID-19 was higher among those with preexisting comorbid conditions. One study showed it to be $10.5 \%$ for cardiovascular disease, $7.3 \%$ for diabetes, $6.3 \%$ for chronic respiratory disease, $6.0 \%$ for hypertension, and $5.6 \%$ for cancer. ${ }^{51}$ The CFR was higher in those cases aged 80 years and above (8.0\%) and among critical COVID-19 cases (49.0\%). The overall case-fatality rate (CFR) of COVID-19 was 2 to $3 \%{ }^{43}$

\section{MANAGEMENT OF COVID-19}

Since by this time, there are no approved treatments for this infection, prevention is the key.

\section{Nonspecific protection from viral infection}

Most viral infections are limited by defenses that are antigen nonspecific. Nonspecific defenses act sooner than specific defenses. Some are always in place (anatomic barriers, nonspecific inhibitors, and phagocytic cells); others are evoked by the infection (fever, inflammation, and interferon). Besides, following measures could be taken by a person for prevention of almost any viral infection. ${ }^{50}$

Resting and avoiding overexertion

Drinking enough water

Avoiding smoking and smoky areas

Taking acetaminophen, ibuprofen, or naproxen for pain and fever

Using a clean humidifier or cool mist vaporizer

Regular hand washing

Covering mouth and nose when coughing and sneezing

Thoroughly cooking meat and eggs

Avoiding close contact with anyone showing symptoms of respiratory illness such as coughing and sneezing

Safe waste management and environmental cleaning

Avoiding unnecessary gatherings and non-essential travel

Preventing COVID-1952,53

Cleaning and disinfecting frequently touched equipment, objects, and surfaces using a regular household cleaning spray or wipe.

Regularly washing hands with alcohol-based hand sanitizer containing at least $60 \%$ alcohol or soaps.

Covering mouth and nose with a tissue when coughing and sneezing, then throwing the tissue in trash or use the inside of your elbow and then use sanitizer.

Avoiding frequent touching of eyes, nose and mouth with unwashed hands as they are the most exposed site for catching the infection.

\section{Staying home when sick}

Using Facemask: Center for disease control (CDC) recommends facemasks to those people who show symptoms of COVID-19 to help prevent the spread of the disease to others. Otherwise, CDC does not recommend that healthy people wear a facemask to protect themselves from COVID-19 when indoor. CDC reminds basic measures such as hand washing, using disinfectant solutions, avoiding contact with patients in order to prevent the spread of viruses by droplets. Everyone should wear a mask in public settings and when around people who 
don't live in one's household, especially when other social distancing measures are difficult to maintain. Masks should not be placed on young children under age 2 , anyone who has trouble breathing, or is unconscious, incapacitated or otherwise unable to remove the mask without assistance. It is good to keep 6 feet distance between two individuals and the mask is not a substitute for social distancing.

Those with multiple comorbidities, weakened immune systems should stay more vigilant. They should be asked to practice cough hygiene by coughing in sleeve/tissue rather than hands and practice hand hygiene frequently every 15 $20 \mathrm{~min}$, minimize the time spent outside and practice social distancing and Self-isolating.

However, several properties of this virus make prevention difficult namely, non-specific features of the disease, the infectivity even before onset of symptoms in the incubation period, transmission from asymptomatic people, long incubation period, tropism for mucosal surfaces such as the conjunctiva, prolonged duration of the illness and transmission even after clinical recovery. ${ }^{54}$

Medical staffs, especially those who come directly in contact with the patients are recommended to wear masks, gowns, eye protection and gloves. Further, applying triage, following correct infection control measures, isolating the cases and contact tracing are key to limit the further spreading of the virus in clinics and hospitals. ${ }^{55}$

\section{Prevention at Community level ${ }^{56}$}

To prevent large-scale community transmission, following measures are to be taken into account listing view:

Educating the public to recognize unusual symptoms

Mitigating social gatherings

Closing school temporarily

Close monitoring of symptomatic individuals

Setting up temperature check or scanning at airports and borders to identify the suspected cases.

Continuing research to trace the source of the outbreak and provide evidence for future outbreak.

Strengthening public health surveillance system coupled with rapid diagnostic testing.

\section{Quarantine/Isolation and Health-care workers ${ }^{57,58}$}

If there is a confirmed case of COVID-19, it should be isolated. Isolation, by definition, separates sick people with a contagious disease from people who are not sick. Ideally, isolation requires hospitalization. The person must be placed in a single patient room with negative air pressure - a minimum of six air changes per hour. Exhausted air has to be filtered through high efficiency particulate air (HEPA) and medical personnel entering the room should wear personal protective equipment (PPE) comprising of gloves, gown, disposable N95, and eye protection. Indeed, isolation is still the most effective method for containing the spread of COVID-19.

Patients can be discharged from isolation once they are afebrile for at least $3 \mathrm{~d}$ and have two consecutive negative molecular tests at $1 \mathrm{~d}$ sampling interval. However, negative molecular test is not a prerequisite for discharge. This recommendation is definition from pandemic flu where patients were asked to resume work/school once afebrile for $24 \mathrm{~h}$ or by day $7^{\text {th }}$ of illness.

If there is suspected case, a 14-day quarantine should be done. Quarantine by defintion separates and restricts the movement of people who were exposed to a contagious disease to see if they become sick. These people may have been exposed to a disease and do not know it, or they may have the disease but do not show symptoms.

If symptoms of a suspected case is only mild, homequarantine is recommended. The ventilation at home should be good with sunlight to allow for destruction of virus. Patients should be asked to wear a simple surgical mask and practice cough hygiene. Caregivers should be asked to wear a surgical mask when in the same room as patient and use hand hygiene every 15-20 min. If more than one patients are there, they should be placed in separate rooms or not cohorted together. Negative pressure rooms are not generally needed. The rooms and surfaces and equipment should undergo regular decontamination preferably with sodium hypochlorite.

Healthcare workers should be provided with fit-tested N95 respirators and protective suits and goggles. Airborne transmission precautions should be taken during aerosol generating procedures such as intubation, suction and tracheostomies. All contacts including healthcare workers should be monitored for development of symptoms of COVID-19. They should use personal protective equipment (PPE) to avoid direct contact with patients' blood, bodyfluids, secretions (including respiratory secretions), and non-intact skin.

\section{Antiviral and immunomodulatory treatment}

Currently,neither there is any universally accepted registered drug to treat COVID-19 nor there is any any FDA approved vaccine. ${ }^{59-61}$ It was not available to MERSCoV and SARS-CoV too. ${ }^{62}$ There are however few drugs with anti-SARS-CoV-2 activities viz., Remdesivir and Hydroxychloroquine.

Remdesivir is a broad spectrum anti-RNA drug developed originally for Ebola virus. Being Nucleotide analogue, it gets incorporated into a nascent viral RNA chain and subsequently causes its premature chain termination. It has demonstrated in-vitro and in-vivo efficacy against SARS-CoV-2. ${ }^{63,64}$ Again, an American study exploring its effectiveness in MERS-CoV mice modals, showed it reduces viral load and improves lung functions. ${ }^{65}$ Thus, it has demonstrated a broad-spectrum action against different 
zoonotic epidemics caused by Corona viruses. It has even be claimed that it was successfully used in several COVID-19 patients in China. ${ }^{66}$

Hydroxychloroquine Sulfate and Chloroquine Phosphate are normally used in Malaria, Extraintestinal Amoebiasis, Autoimmune diseases eg, SLE. Chloroquine has also shown in vitro and in vivo (animal models) effectiveness against SARS-CoV and avian influenza. ${ }^{67-69}$ United States Food and Drug Administration (USFDA) has provided an Emergency Use Authorization (EUA) use of these drugs to the patients with COVID-19. ${ }^{70}$ Its antiviral efficacy seems to be explained by an increase in endosomal $\mathrm{pH}$ which is necessary for fusion between the virus and the host cell; they also seem to interfere with the ACE2 cell receptor and have immune-modulatory activity. Hydroxychloroquine is more potent than Chloroquine in inhibiting SARS-CoV-2. These antimalarial medications may act synergistically with Macrolides too (e.g. Azithromycin). ${ }^{71}$

Other treatment options with no proven efficacy are Lopinavir/Ritonavir, Arbidol, and Shufengjiedu Capsule (A Traditional Chinese Medicine), Anti-HIV Drugs, Pegylated Interferon Andibavirin, IV Immunoglobulin, Interferons, Ribavirin and Umifenovir. ${ }^{72}$

\section{Treatment of complications ${ }^{74}$}

Treatments of COVID-19 complications are essentially supportive and symptomatic as written below

1. Early administration of antipyretic and analgesic

2.Maintenance of hydration / conservative fluid management. There is a high risk of acute kidney injury with hypovolemia

\section{Close monitoring for signs of deterioration}

4. Administering appropriate empirical antibiotics and antifungals immediately (within an hour) of sepsis identification.

5. Early Anticoagulation therapy when the D-dimer value is four times higher than normal.

6. Probiotics to patients to maintain the equilibrium of the gut microflora and ameliorate gastrointestinal symptoms to prevent secondary bacterial infection.

7. Early institution of respiratory (Mechanical ventilation/ Extracorporeal membrane oxygenation), renal, cardiovascular as well as psychological/psychiatric support.

8. Glucocorticoids should be considered in severe COVID-19 to prevent ARDS progression. ${ }^{73}$

\section{Vaccine and probable future Treatment}

Based on the consolidated clinical data, derived from five independent studies of 27 patients, it is suggested that Passive immunotherapy in the form of convalescent plasma transfusion (CPT), in addition to antiviral/antimicrobial drugs, could be an effective therapeutic option with promising evidence on safety, improvement of clinical symptoms, and reduced mortality. ${ }^{75}$

Public health experts are hoping for one or several vaccines to combat COVID-19 by early 2021. Now, there are close to 200 vaccines for COVID-19 under study, and many of them have moved to phase III human studies which is the final step to prove if the vaccine is safe and effective before it can get FDA approval. Main companies involved in the vaccine-development programs are AstraZeneca, Janssen (Johnson \& Johnson), Moderna, Novavax, Pfizer and Sanofi/GSK. The FDA has also said that it could issue an emergency use authorization (EUA) for a COVID-19 vaccine on a case-by-case basis. ${ }^{76-78}$

\section{Other potentially interesting but purely speculative options include:}

Predicting mutation trends in Corona viruses along with surveillance systems which have been proven to be pivotal in controlling current and future outbreaks.

Monoclonal antibodies and immune-stimulants including those acting on the Th1-mediated inflammatory cascade capable of neutralizing variant strains including SARSCoV-2. Few examples are:

- Canakinumab (a human monoclonal antibody targeting IL- 1 b) ${ }^{79}$

- Roflumilast (a selective long-acting inhibitor of the enzyme phosphodiesterase-4) which is already used to control Neutrophilic inflammation in patients with COPD. ${ }^{80}$

- Monoclonal antibodies against the S1 domain of the S protein. ${ }^{81}$

\section{Effect of COVID-19 on other aspects of life}

The Corona virus has already affected the world economy and damaged the global market. This new virus outbreak has challenged the economic, medical and public health infrastructure of the world. Corona is an invasion of reality and a slap in the face to the proud world. At the same time, it can be a factor of social solidarity and empathy and cooperation, morality and rationality. Today's message to mankind is that there is no other way but peaceful interaction and coexistence with one's fellow man and the environment. Thus, along with all the negative consequences, Corona can be strengthen world peace and unity and be the beginning of a peaceful and virtuous lifestyle. ${ }^{82}$

\section{Could SARS-CoV-2/COVID-19 simply fade away?}

SARS-CoV-2 has progressively invaded almost every corner of the world. Two previous highly pathogenic outbreaks of Coronavirus infections have occurred in the last decades: SARS-CoV outbreak which started in China in 2003 and MERS-CoV first was identified in Saudi Arabia in 2012.

By spontaneous genetic attenuation through mutation of RNA viruses, the virus may fade away. While almost every country faces this COVID-19 wave, options are that the 
virus drops its lethality over time and may even go temporal extinction over the course of years ahead. ${ }^{83}$

\section{CONCLUSION}

COVID-19 has drastically changed normal human life. It has affected every facet of human life viz., cultural, economic, political, educational, environmental, psychological, religious, and jurisprudential probably like no other human disease has ever done before. And yet answer to the question "when will the world get rid of this deadly virus?" is still unknown. As the death toll and morbidity by the disease is rising every second, time alone will tell its whole story.
Today as the future of COVID-19 depends upon host adaptation, viral evolution, infectivity, transmissibility and pathogenicity, most eyes are eagerly laid against early development and approval of vaccine or spontaneous genetic attenuation through mutation.

Learning from the present pandemic, on one hand, we should take comprehensive measures to prevent future outbreaks of zoonotic origin and on the other, people should stop spreading myths and false information about the disease and try to allay panic and anxiety among the public.

\section{REFERENCES}

1. Lu H, Stratton CW, Tang Y-W. Outbreak of pneumonia of unknown etiology in Wuhan, China: The mystery and the miracle. J Med Virol. 2020; 92: 401-2.

2. Hui DS, I Azhar E, Madani TA, et al. The continuing 2019-nCoV epidemic threat of novel coronaviruses to global health - The latest 2019 novel coronavirus outbreak in Wuhan, China. International journal of infectious diseases: IJID: official publication of the International Society for Infectious Diseases 2020; 91: 264-6.

3. Rosselli D, Yucuma D, Rodriguez-Morales A, et al. Could SARS-CoV-2/ COVID-19 simply fade away? Le Infez Med Riv Period di eziologia, Epidemiol diagnostica, Clin e Ter delle Patol Infett 2020; 28: in press.

4. Huang $C$, Wang $Y, L i X$, et al. Clinical features of patients infected with 2019 novel coronavirus in Wuhan, China. Lancet (London, England) 2020; 395: 497-506.

5. Gorbalenya AE, Baker SC, Baric RS, et al. Severe acute respiratory syndrome-related coronavirus: The species and its viruses a statement of the Coronavirus Study Group. bioRxiv 2020; 2020.02.07.937862.

6. Burki TK. Coronavirus in China. The Lancet. Respiratory medicine. 2020; 8: 238.

7. Rothe C, Schunk M, Sothmann P, et al. Transmission of 2019-nCoV Infection from an Asymptomatic Contact in Germany. N Engl J Med. 2020; 382: 970-1.

8. Chan-Yeung M, Xu R-H. SARS: epidemiology. Respirology. 2003; 8 Suppl: S9-14.

9. Middle East respiratory syndrome coronavirus (MERS-CoV)https:// www.who.int/emergencies/mers-cov/en/ (2019).

10. Burrell C, Howard C MFF. White's medical virology. $5^{\text {th }}$ ed. United States: Academic Press, 2016.

11. Bárcena M, Oostergetel GT, Bartelink W, et al. Cryo-electron tomography of mouse hepatitis virus: Insights into the structure of the coronavirion. Proc Natl Acad Sci U S A. 2009; 106: 582-7.

12. Neuman BW, Adair BD, Yoshioka C, et al. Supramolecular architecture of severe acute respiratory syndrome coronavirus revealed by electron cryomicroscopy. J Virol. 2006; 80: 7918-28.

13. Klausegger A, Strobl B, Regl G, et al. Identification of a coronavirus hemagglutinin-esterase with a substrate specificity different from those of influenza C virus and bovine coronavirus. J Virol. 1999; 73: 3737-43.

14. Jahangir M. Coronavirus (COVID-19): History, Current Knowledge and Pipeline Medications. Int J Pharm Pharmacol. 2020; 4: 1-9.

15. Peiris JSM, Guan Y, Yuen KY. Severe acute respiratory syndrome. Nat Med. 2004; 10: S88-97.

16. Zaki AM, van Boheemen S, Bestebroer TM, et al. Isolation of a novel coronavirus from a man with pneumonia in Saudi Arabia. N Engl J Med. 2012; 367: 1814-20.

17. Lu R, Zhao X, Li J, et al. Genomic characterisation and epidemiology of 2019 novel coronavirus: implications for virus origins and receptor binding. Lancet (London, England) 2020; 395: 565-74.

18. Luby SP, Gurley ES, Hossain MJ. Transmission of human infection with Nipah virus. Clin Infect Dis an Off Publ Infect Dis Soc Am. 2009; 49: 1743-8.

19. Chen N, Zhou M, Dong X, et al. Epidemiological and clinical characteristics of 99 cases of 2019 novel coronavirus pneumonia in Wuhan, China: a descriptive study. Lancet (London, England) 2020; 395: 507-13.

20. Cho P, Boost M. COVID 19-An eye on the virus. Cont Lens Anterior Eye. 2020; 43: 313-4.

21. Kampf G, Todt D, Pfaender S, et al. Persistence of coronaviruses on inanimate surfaces and their inactivation with biocidal agents. $J$ Hosp Infect. 2020; 104: 246-51.

22. Li Q, Guan X, Wu P, et al. Early Transmission Dynamics in Wuhan, China, of Novel Coronavirus-Infected Pneumonia. N Engl J Med. 2020; 382: 1199-1207.

23. Liu $Y$, Gayle AA, Wilder-Smith A, et al. The reproductive number of COVID-19 is higher compared to SARS coronavirus. J Travel Med. 27. Epub ahead of print March 2020. DOI: 10.1093/jtm/taaa021.

24. Chang $D, X u H$, Rebaza A, et al. Protecting health-care workers from subclinical coronavirus infection. The Lancet. Respiratory medicine 2020; 8: e13.

25. Xu X, Chen P, Wang J, et al. Evolution of the novel coronavirus from the ongoing Wuhan outbreak and modeling of its spike protein for risk of human transmission. Science China. Life sciences. 2020; 63: 457-60.

26. Kuba $\mathrm{K}$, Imai $\mathrm{Y}, \mathrm{Rao} \mathrm{S}$, et al. A crucial role of angiotensin converting enzyme 2 (ACE2) in SARS coronavirus-induced lung injury. Nat Med. 2005; 11: 875-879.

27. Chen H, Guo J, Wang C, et al. Clinical characteristics and intrauterine vertical transmission potential of COVID-19 infection in nine pregnant women: a retrospective review of medical records. Lancet (London, England) 2020; 395: 809-15.

28. Shen K-L, Yang Y-H. Diagnosis and treatment of 2019 novel coronavirus infection in children: a pressing issue. World journal of pediatrics WJP. 2020; 16: 219-21.

29. Singhal T. A Review of Coronavirus Disease-2019 (COVID-19). Indian J Pediatr. 2020; 87: 281-286. 
30. Cheng ZJ, Shan J. 2019 Novel coronavirus: where we are and what we know. Infection. 2020; 48: 155-63.

31. Yang $X, Y u Y, X u J$, et al. Clinical course and outcomes of critically ill patients with SARS-CoV-2 pneumonia in Wuhan, China: a singlecentered, retrospective, observational study. Lancet Respir Med. 2020; 8: 475-81.

32. Wang D, Hu B, Hu C, et al. Clinical Characteristics of 138 Hospitalized Patients With 2019 Novel Coronavirus-Infected Pneumonia in Wuhan, China. JAMA. 2020; 323: 1061-9.

33. Zhu N, Zhang D, Wang W, et al. A Novel Coronavirus from Patients with Pneumonia in China, 2019. N Eng/ J Med. 2020; 382: 727-33.

34. Desforges $M$, Le Coupanec A, Dubeau P, et al. Human Coronaviruses and Other Respiratory Viruses: Underestimated Opportunistic Pathogens of the Central Nervous System? Viruses. 2019; 12: 14.

35. Xu Z, Shi L, Wang Y, et al. Pathological findings of COVID-19 associated with acute respiratory distress syndrome. The Lancet. Respiratory medicine 2020; 8: 420-2.

36. He F, Deng Y, Li W. Coronavirus disease 2019: What we know? J Med Virol. 2020; 92: 719-25.

37. Khunti $K$, Singh AK, Pareek $M$, et al. Is ethnicity linked to incidence or outcomes of covid-19? BMJ. 2020; 369: m1548.

38. Pascarella G, Strumia A, Piliego C, et al. COVID-19 diagnosis and management: a comprehensive review. J Intern Med. 2020; 288: 192-206.

39. Lan L, Xu D, Ye G, et al. Positive RT-PCR Test Results in Patients Recovered From COVID-19. JAMA. 2020; 323: 1502-1503.

40. Laureano AFS, Riboldi M. The different tests for the diagnosis of COVID-19 - A review in Brazil so far. JBRA Assist Reprod. 2020; 24 : 340-6.

41. Li M. Chest CT features and their role in COVID-19. Radiol Infect Dis. (Beijing, China) 2020; 7: 51-54.

42. Mohammadi M, Meskini M, do Nascimento Pinto AL. 2019 Novel coronavirus (COVID-19) overview. J Public Health (Bangkok). Epub ahead of print 2020. DOI: 10.1007/s10389-020-01258-3.

43. Abebe EC, Dejenie TA, Shiferaw MY, et al. The newly emerged COVID-19 disease: a systemic review. Virol J. 2020; 17: 96.

44. Chan JF-W, Yuan S, Kok K-H, et al. A familial cluster of pneumonia associated with the 2019 novel coronavirus indicating person-toperson transmission: a study of a family cluster. Lancet. (London, England) 2020; 395: 514-23.

45. Young BE, Ong SWX, Kalimuddin S, et al. Epidemiologic Features and Clinical Course of Patients Infected With SARS-CoV-2 in Singapore. JAMA. 2020; 323: 1488-94.

46. Zhang W, Du R-H, Li B, et al. Molecular and serological investigation of 2019-nCoV infected patients: implication of multiple shedding routes. Emerg Microbes Infect. 2020; 9: 386-9.

47. Tersalvi G, Vicenzi M, Calabretta D, et al. Elevated Troponin in Patients With Coronavirus Disease 2019: Possible Mechanisms. J Card Fail. 2020; 26: 470-75.

48. Hoang A, Chorath K, Moreira A, et al. COVID-19 in 7780 pediatric patients: A systematic review. E Clinical Medicine. 2020; 24: 100433.

49. Bernheim A, Mei X, Huang $M$, et al. Chest CT Findings in Coronavirus Disease-19 (COVID-19): Relationship to Duration of Infection. Radiology. 2020; 295: 200463.

50. Peeri NC, Shrestha N, Rahman MS, et al. The SARS, MERS and novel coronavirus (COVID-19) epidemics, the newest and biggest global health threats: what lessons have we learned? Int J Epidemiol. 2020; 49: 717-26.

51. Wu Z, McGoogan JM. Characteristics of and Important Lessons From the Coronavirus Disease 2019 (COVID-19) Outbreak in China: Summary of a Report of 72314 Cases From the Chinese Center for Disease Control and Prevention. JAMA. 2020; 323: 1239-42.
52. Wax RS, Christian MD. Practical recommendations for critical care and anesthesiology teams caring for novel coronavirus (2019-nCoV) patients. Can J Anaesth. 2020; 67: 568-76.

53. Control C for disease. Coronavirus Disease 2019 (COVID-19); How to Protect Yourself \& Othershttps://www.cdc.gov/coronavirus/2019ncov/prevent-getting-sick/prevention.html (2020).

54. Jin Y-H, Cai L, Cheng ZS, et al. A rapid advice guideline for the diagnosis and treatment of 2019 novel coronavirus (2019-nCoV) infected pneumonia (standard version). Mil Med Res. 2020; 7: 4.

55. Chan JFW, Lau SKP, To KKW, et al. Middle East respiratory syndrome coronavirus: another zoonotic betacoronavirus causing SARS-like disease. Clin Microbiol Rev. 2015; 28: 465-522.

56. Heymann DL, Shindo N. COVID-19: what is next for public health? Lancet (London, England) 2020; 395: 542-5.

57. ZHOU G, Chi C. A model simulation study on effects of intervention measures in Wuhan COVID-19 epidemic. 2020. Epub ahead of print. 18 February 2020. DOI: 10.1101/2020.02.14.20023168.

58. What is the difference between isolation and quarantine?https:// www.hhs.gov/answers/public-health-and-safety/what-is-thedifference-between-isolation-and-quarantine/index.html (2020).

59. Prompetchara E, Ketloy C, Palaga T. Immune responses in COVID-19 and potential vaccines: Lessons learned from SARS and MERS epidemic. Asian Pacific J allergy Immunol. 2020;38:1-9.

60. Pang J, Wang MX, Ang IYH, et al. Potential Rapid Diagnostics, Vaccine and Therapeutics for 2019 Novel Coronavirus (2019-nCoV): A Systematic Review. J Clin Med. Epub ahead of print February 2020. DOI: $10.3390 / \mathrm{jcm} 9030623$.

61. Shanmugaraj B, Malla A, Phoolcharoen W. Emergence of Novel Coronavirus 2019-nCoV: Need for Rapid Vaccine and Biologics Development. Pathog (Basel, Switzerland); 9. Epub ahead of print February 2020. DOI: 10.3390/pathogens9020148.

62. Tang JW, Tambyah PA, Hui DSC. Emergence of a novel coronavirus causing respiratory illness from Wuhan, China. The Journal of infection. 2020; 80: 350-71.

63. Wang $M$, Cao $R$, Zhang $L$, et al. Remdesivir and chloroquine effectively inhibit the recently emerged novel coronavirus (2019-nCoV) in vitro. Cell research. 2020; 30: 269-71.

64. Wit E, Feldmann F, Cronin J, et al. Prophylactic and therapeutic remdesivir (GS-5734) treatment in the rhesus macaque model of MERS-CoV infection. Proc Natl Acad Sci. 2020; 117: 201922083.

65. Sheahan TP, Sims AC, Leist SR, et al. Comparative therapeutic efficacy of remdesivir and combination lopinavir, ritonavir, and interferon beta against MERS-CoV. Nat Commun. 2020; 11: 222.

66. Al-Tawfiq JA, Al-Homoud AH, Memish ZA. Remdesivir as a possible therapeutic option for the COVID-19. Travel medicine and infectious disease. 2020; 34: 101615.

67. Savarino A, Di Trani L, Donatelli I, et al. New insights into the antiviral effects of chloroquine. Lancet Infect Dis. 2006; 6: 67-9.

68. Vincent MJ, Bergeron E, Benjannet $S$, et al. Chloroquine is a potent inhibitor of SARS coronavirus infection and spread. Virol J. 2005;2: 69.

69. Yan $Y$, Zou Z, Sun $Y$, et al. Anti-malaria drug chloroquine is highly effective in treating avian influenza A H5N1 virus infection in an animal model. Cell research. 2013; 23: 300-2.

70. Coronavirus (COVID-19) Update: Daily Roundup March 30, 2020https://www.fda.gov/news-events/press-announcements/ coronavirus-covid-19-update-daily-roundup-march-30-2020.

71. Adhikari SP, Meng S, Wu Y-J, et al. Epidemiology, causes, clinical manifestation and diagnosis, prevention and control of coronavirus disease (COVID-19) during the early outbreak period: a scoping review. Infect Dis poverty 2020; 9: 29.

72. Rabby MII. Current Drugs with Potential for Treatment of COVID-19: A Literature Review. J Pharm Pharm Sci a Publ Can Soc Pharm Sci Soc Can des Sci Pharm. 2020; 23: 58-64. 
73. So C, Ro S, Murakami M, et al. High-dose, short-term corticosteroids for ARDS caused by COVID-19: a case series. Respirology case reports. 2020; 8: e00596.

74. Cascella M, Rajnik M, Cuomo A, et al. Features, Evaluation, and Treatment of Coronavirus (COVID-19). Treasure Island (FL), 2020.

75. Rajendran K, Krishnasamy N, Rangarajan J, et al. Convalescent plasma transfusion for the treatment of COVID-19: Systematic review. J Med Virol. Epub ahead of print May 2020. DOI: 10.1002/jmv.25961.

76. Le T, Andreadakis Z, et al. The COVID-19 vaccine development landscape. Nat Rev Drug Discov. Epub ahead of print 9 April 2020. DOI: 10.1038/d41573-020-00073-5.

77. Funk CD, Laferrière $C$, Ardakani $A$. A Snapshot of the Global Race for Vaccines Targeting SARS-CoV-2 and the COVID-19 Pandemic. Frontiers in Pharmacology. 2020; 11: 937.

78. Doheny K. COVID-19 Vaccine: Latest Updateshttps://www.webmd. com/lung/news/20200610/covid-19-latest-updates (2020, accessed 13 September 2020).
79. Chakraborty A, Tannenbaum S, Rordorf C, et al. Pharmacokinetic and pharmacodynamic properties of canakinumab, a human antiinterleukin-1 $\beta$ monoclonal antibody. Clin Pharmacokinet. 2012; 51: e1-18.

80. Wedzicha JA, Calverley PM, Rabe KF. Roflumilast: a review of its use in the treatment of COPD. Int J Chron Obstruct Pulmon Dis. 2016; 11: 81-90.

81. Sui J, Li W, Murakami A, et al. Potent neutralization of severe acute respiratory syndrome (SARS) coronavirus by a human mAb to S1 protein that blocks receptor association. Proc Natl Acad Sci U S A. 2004; 101: 2536-41.

82. Nicola M, Alsafi Z, Sohrabi C, et al. The socio-economic implications of the coronavirus pandemic (COVID-19): A review. Int J Surg. 2020; 78: 185-93.

83. Molecular evolution of the SARS coronavirus during the course of the SARS epidemic in China. Science. 2004; 303: 1666-9. 